\title{
Partição gástrica para o tratamento paliativo de pacientes com tumores gástricos distais obstrutivos e irresecáveis
}

\author{
Gastric partitioning for the palliative treatment of patients with unresectable \\ distal gastric cancer with gastric outlet obstruction
}

\author{
Brian Guilherme Monteiro Marta Coimbra ${ }^{1}$, Marcus Fernando Kodama Pertille Ramos ${ }^{2}$, \\ Osmar Kenji Yagi ${ }^{3}$, Carlos Eduardo Jacob ${ }^{4}$, Ivan Cecconello ${ }^{5}$, Bruno Zilbertein ${ }^{6}$
}

Coimbra BGMM, Ramos MFKP, Yagi OK, Jacob CE, Cecconello I, Zilberstein B. Partição gástrica para o tratamento paliativo de pacientes com tumores gástricos distais obstrutivos e irresecáveis / Gastric partitioning for the palliative treatment of patients with unresectable distal gastric cancer with gastric outlet obstruction. Rev Med (São Paulo). 2015 jan.-mar.;94(1):21-8.

\begin{abstract}
RESUMO: O câncer gástrico é uma afecção que ainda se mostra muito prevalente. Como agravante, a maioria dos pacientes apresenta-se em estágios avançados da doença, frequentemente com tumores distais obstrutivos e irressecáveis, demandando tratamento paliativo, cujo objetivo é dar ao doente maior qualidade de vida. Dentre as opções terapêuticas, tradicionalmente se opta pela gastroenteroanastomose. Entretanto, devido às altas taxas de complicações desse procedimento, passou-se a empregar a técnica da partição gástrica. Frente aos resultados em estudos comparativos entre essas duas técnicas, nosso serviço passou a realizar a partição gástrica em casos de pacientes portadores de câncer gástrico avançado distal obstrutivo e irressecável, e analisamos retrospectivamente os resultados do emprego dessa técnica em 29 pacientes, em termos de sobrevida e qualidade de
\end{abstract}

vida. Toda a população se apresentava com ECOG performance status de 0,1 ou 2 e $53,9 \%$ dos doentes apresentavam, sob a classificação de obstrução gástrica de GOOSS, graus 0 ou 1. A duração operatória média foi de 169 minutos. No pós-operatório, sob a classificação de complicações de Clavien-Dindo, houve 4 pacientes em grau II e 2 em grau V (dois óbitos, correspondendo a uma mortalidade operatória de 6,9\%). O tempo médio para se atingir GOOSS 2 foi de 5 dias e, ao longo do seguimento, $96,15 \%$ da população atingiu GOOSS 2 ou 3. A sobrevida média foi de 213 dias. Considerando os resultados satisfatórios obtidos, a técnica de partição gástrica é efetiva e segura, proporcionando sobrevida, manutenção de dieta via oral e qualidade de vida para os pacientes.

Descritores: Cirurgia; Neoplasias gástricas; Cuidados paliativos; Obstrução da saída gástrica

$2^{\circ}$ lugar Prêmio Oswaldo Cruz - Área: Cirúrgica no $33^{\circ}$ COMU - Congresso Médico Universitário da FMUSP, SP, 31 de out. a 02 de nov. de 2014.

1. Acadêmico de Medicina da Faculdade de Medicina da Universidade de São Paulo. E-mail: brian_coimbra_2007@hotmail.com.

2. Orientador, Departamento de Gastroenterologia, Disciplina de Cirurgia do Aparelho Digestivo da Faculdade de Medicina da Universidade de São Paulo; ICESP - Instituto do Câncer do Estado de São Paulo. E-mail: marcuskodama@hotmail.com.

3. Orientador, Departamento de Gastroenterologia, Disciplina de Cirurgia do Aparelho Digestivo da Faculdade de Medicina da Universidade de São Paulo; ICESP - Instituto do Câncer do Estado de São Paulo. E-mail: okyagi@uol.com.

4. Orientador, Departamento de Gastroenterologia, Disciplina de Cirurgia do Aparelho Digestivo da Faculdade de Medicina da Universidade de São Paulo. E-mail: cejacob@uol.com.br.

5. Orientador, Professor Titular do Departamento de Gastroenterologia, Disciplina de Cirurgia do Aparelho Digestivo da Faculdade de Medicina da Universidade de São Paulo. E-mail: icecconello@hotmail.com.

6. Orientador, Professor Livre Docente do Departamento de Gastroenterologia, Disciplina de Cirurgia do Aparelho Digestivo da Faculdade de Medicina da Universidade de São Paulo.E-mail: brunozilb@uol.com.br.

Endereço para correspondência: Depto. de Gastroenterologia do HCFMUSP. Rua Ovídio Pires de Campos, 225 - 9o andar. Cerqueira César, São Paulo, SP, Brasil. CEP: 05403-010. 
ABSTRACT: Gastric cancer is still a very prevalent decease. Most patients show up in latter stages of the decease, frequently with unresectable distal tumor and gastric outlet obstruction, in need of palliative care, which main purpose is to provide the patient a better quality of life. Among the therapeutic alternatives, gastrojejunostomy comes as a traditional choice. However, because of the high complication rates of this procedure, the gastric partitioning surgery emerged as an option. Given the relevant results presented in comparative studies between these two techniques, our service started performing the gastric partitioning surgery for the treatment of gastric outlet obstruction in patients with unresectable distal gastric cancer. We analyzed retrospectively the results in terms of survival rate and quality of

\section{INTRODUÇÃO}

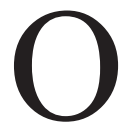
câncer ocupa uma posição de destaque dentre as causas de morbi-mortalidade da população brasileira e esta situação agravar-se-á nas próximas décadas, com o aumento da incidência da doença associado ao envelhecimento da população.

Outrora o segundo tipo mais comum de câncer no mundo (atualmente é o quarto), o câncer gástrico teve sua incidência diminuída nos últimos 50 anos. Essa queda se deve em parte à disseminação do uso de refrigeradores, que alterou a relação humana com os alimentos (aumentando o consumo de frutas e vegetais frescos, diminuindo a ingestão de sal - então largamente usado como conservante - e proporcionando queda na contaminação da comida por componentes carcinogênicos emergentes da decomposição de produtos como a carne não refrigerada). Outros fatores contribuintes com esse declínio incluem, por exemplo, as menores taxas de infecção crônica por Helicobacter pylori, graças à evolução nos sistemas de saneamento e no desenvolvimento de antibióticos, e a intensificação na pesquisa preventiva de câncer (screening ou rastreamento) em vários países ${ }^{1}$.

Geograficamente, a incidência da doença se espalha de modo variado ao redor do mundo, com taxas mais reduzidas na América do Norte e mais elevadas na Ásia e partes da América do Sul (incluindo o Brasil). Os maiores índices de morte relacionada a esse tipo de câncer também acompanham geograficamente as taxas elevadas de ocorrência da doença ${ }^{2}$.

Embora menos freqüente, é ainda muito prevalente e se encontra entre as três primeiras causas mais comuns de morte relacionada a câncer em âmbito mundial, consistindo na segunda principal causa de morte por câncer no sexo masculino e terceira no sexo feminino (Parkin et al., 2005). Além disso, a taxa de incidência é cerca de duas vezes mais alta no sexo masculino do que no feminino. No ano de 2012, estimou-se, para o Brasil, 12.670 casos novos de câncer do estômago em homens e 7.420 em mulheres. Esses valores correspondem a um risco estimado de 13 casos novos a life. ECOG performance status of all patients were 0,1 or 2 and $53,9 \%$ presented GOOSS grades 0 or 1 . Mean operation time was 169 minutes. In the postoperative period, there were 4 grade II Clavien-Dindo patients and 2 grade V (two deaths, meaning an operative mortality of $6,9 \%$ ). Mean time to achieve GOOSS grade 2 was 5 days and, throughout the follow up, $96,15 \%$ of the patients achieved GOOSS grades 2 or 3 . Mean survival rate was 213 days. Based on the obtained satisfactory results, the gastric portioning surgery is safe and effective, allowing the patients pertinent survival rate, oral food intake and quality of life.

Descritores: Surgery; Stomach neoplasms; Palliative care; Gastric Outlet obstruction.

cada 100 mil homens e 7 a cada 100 mil mulheres. Sem considerar os tumores de pele não melanoma, o câncer do estômago em homens é o segundo mais frequente nas regiões Norte $(11 / 100 \mathrm{mil})$ e Nordeste $(9 / 100 \mathrm{mil})$ e o quarto nas regiões Sul (16/100 mil), Sudeste (15/100 mil) e Centro-Oeste (14/100 mil). Para as mulheres, ocupa a quarta posição na região Norte $(6 / 100 \mathrm{mil})$, a quinta na região Centro-Oeste (7/100 mil) e a sexta nas regiões Sudeste (9/100 mil), Sul (8/100 mil) e Nordeste (6/100mil).

Vale mencionar que, na maioria dos países, a maior parte dos pacientes chega em estágios avançados da doença, devido ao fraco rastreamento específico deste tipo de câncer nestes países e porque somente nesta condição tardia é que se acentua a sintomatologia clínica (náusea, disfagia, queda no apetite e perda notável de peso sem auxílio de dieta).

No Brasil, dentre os casos com estadiamento informado, estima-se que mais de $70 \%$ dos casos sejam dos estádios III e IV, e em $87,5 \%$ destes a ressecção do estômago não é possível ( $38^{\circ}$ Gastrão, 2011, p. 95). Dentre os tumores avançados, $54 \%$ se localizam nas regiões antral e pilórica e especificamente quanto ao estadio mais avançado, o estadio IV, em 52\% dos casos a localização do tumor se encontra no terço distal do órgão ${ }^{3,4}$.

Haja vista a relevância dos casos irresecáveis e de localização distal, não é raro encontrar casos de pacientes com doença obstrutiva. Nesses casos, opta-se pelo tratamento paliativo, visando dar aos doentes uma vida digna, com menos dor, o mais próxima do normal e a mais longa possível. Em outras palavras, procura-se aumentar a qualidade de vida dos pacientes através da viabilização da alimentação de forma adequada ou, ao menos, através do alívio da dificuldade alimentar ${ }^{5,6}$.

A abordagem paliativa do doente pode ser realizada através de algumas técnicas cirúrgicas e endoscópicas, a exemplo da gastrojejunoanastomose ${ }^{7}$ (técnica cirúrgica mais tradicional) e das próteses endoscópicas ${ }^{8,9}$. Como o tratamento com próteses, apesar dos resultados iniciais satisfatórios, apresenta alto custo, altas taxas de recidiva dos sintomas obstrutivos e baixa sobrevida dos pacientes, 
este procedimento acaba por ter sua utilização limitada a pacientes com expectativa de vida menor que dois meses e más condições clínicas (sob classificação da Eastern Cooperative Oncology Group - ECOG - de 3 e 4), sendo assim preterido em detrimento do uso da cirurgia de derivação gástrica interna (pouco mais vantajosa, como mostra a tabela a seguir), conforme preconizado por Jeurnink et al. ${ }^{10}$ após realização de estudo randomizado multicêntrico $(\text { SUSTENT Study })^{10}$.

Tabela1. Comparação entre o emprego de prótese e gastrojejunoanastomose no tratamento paliativo de câncer gástrico distal avançado. (Adaptado de: Jeurnink et al. ${ }^{10}$ )

\begin{tabular}{|c|c|c|}
\hline Fatores de Comparação & $\begin{array}{l}\text { Prótese } \\
(\%)\end{array}$ & $\begin{array}{l}\text { Gastrojejunoa- } \\
\text { nastomose (\%) }\end{array}$ \\
\hline Sucesso Técnico & 96 & 99 \\
\hline Sucesso Clínico & 89 & 72 \\
\hline Complicações Precoces & 7 & 4 \\
\hline Complicações Tardias & 18 & 17 \\
\hline Complicações Menores & 9 & 33 \\
\hline $\begin{array}{l}\text { Sintomas Obstrutivos } \\
\text { Persistentes }\end{array}$ & 8 & 9 \\
\hline Reoperações & 8 & 1 \\
\hline $\begin{array}{l}\text { Estadia Hospitalar Média } \\
\text { (dias) }\end{array}$ & 7 & 13 \\
\hline $\begin{array}{l}\text { Sobrevivência Média } \\
\text { (dias) }\end{array}$ & 105 & 164 \\
\hline
\end{tabular}

Assim, a principal modalidade terapêutica não curativa para o tratamento do câncer gástrico obstrutivo é a cirurgia. Sempre que possível, a ressecção do tumor sem linfadenectomia associada dever ser realizada, pois viabiliza maior durabilidade de aceitação da dieta via oral com melhora da qualidade de vida ${ }^{11,12}$. No entanto, muitos pacientes apresentam tumores localmente avançados, que não podem ser ressecados ${ }^{13}$. A incidência de pacientes com câncer gástrico nesta situação varia na literatura entre 5 a $30 \%{ }^{14}$. Nestes casos, os procedimentos de derivação gastro-intestinal podem proporcionar melhora na qualidade de vida, aliviando os sintomas de dificuldade de ingestão oral sem apresentar alto risco cirúrgico.

A derivação gástrica consiste em um procedimento que promove a passagem do conteúdo gástrico para o intestino delgado (duodeno ou jejuno) através de uma anastomose realizada acima de um ponto de obstrução, sem, no entanto, promover sua ressecção. As técnicas de cirurgia de derivação gástrica evoluíram com o passar do tempo. Inicialmente, Wölfer introduziu em 1881 a gastrojejunostomia (Figura 1), técnica largamente empregada por muito tempo, sendo a mais tradicionalmente adotada até os dias atuais. Esta anastomose é realizada na parede posterior do estômago com a primeira alça jejunal que o alcança sem tensão, de maneira látero-lateral e com ampla extensão, podendo ser realizada de maneira manual ou mecânica. O procedimento é simples e de rápida execução, mas seus inconvenientes são a dificuldade de esvaziamento gástrico pela anastomose ${ }^{15}$ (com 10 a $26 \%$ de freqüência referida na literatura), a ocorrência elevada de sangramento tumoral (decorrente da manutenção do contato do tumor com a dieta ingerida) e o risco de nova obstrução pelo crescimento do tumor, que se encontra próximo a anastomose. Assim, as altas taxas de sintomas pós-operatórios e de insatisfação dos pacientes, além das complicações, fizeram com que essa técnica fosse preterida em detrimento de outras, como a de gastro-jejunostomia exclusiva proposta originalmente por Devine ${ }^{16}$, em 1925, que consistia em um método de exclusão antral e divisão completa do estômago acompanhada de uma gastroentero anastomose na câmara gástrica proximal para o manejo de úlceras duodenais difíceis. Posteriormente, Maingot et al. ${ }^{17}$ utilizaram a mesma técnica para o manejo de tumores gástricos distais obstrutivos denominando-o procedimento de Devine. Devido ao risco de rompimento do coto gástrico distal por acúmulo de secreções tumorais, Kaminishi et al. ${ }^{18}$ descreveram em 1997 uma modificação da cirurgia mantendo-se um segmento gástrico pérvio de aproximadamente $3 \mathrm{~cm}$ na pequena curvatura comunicando as duas câmaras gástricas que foram separadas pelo grampeamento horizontal do estômago. Esta comunicação permite o refluxo de conteúdo da câmara distal para a proximal, onde é realizada a anastomose com o intestino delgado. Desta maneira, evita-se o risco de rompimento da câmara gástrica distal, diminui-se o contato entre os alimentos ingeridos e o tumor e ainda viabiliza-se a avaliação endoscópica do tumor através do conduto pérveo que separa as câmaras gástricas. Este procedimento é referido na literatura como exclusão de Devine modificada, bipartição gástrica ou ainda partição gástrica, denominação que adotaremos neste trabalho (Figura 2). 


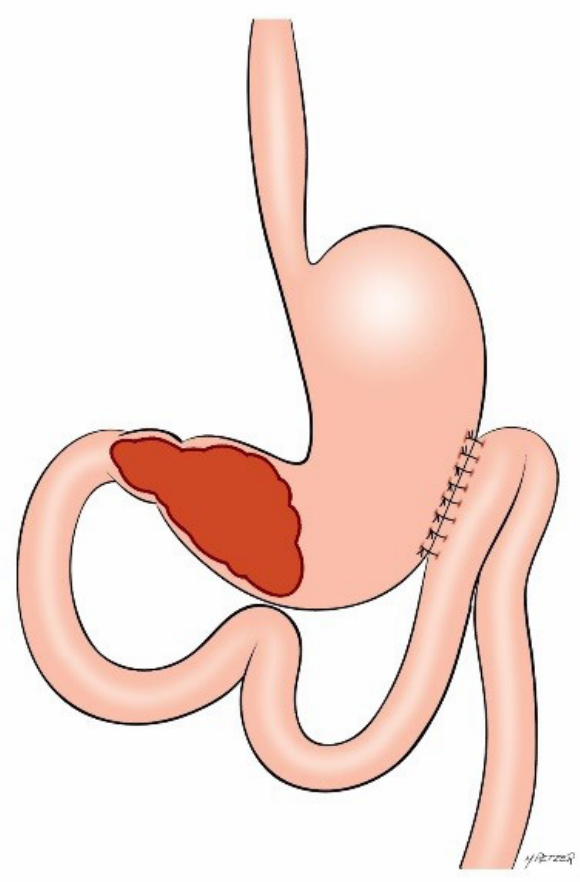

Figura 1. Gastroenteroanastomose proposta por Wölfer

A técnica pode ser realizada por laparoscopia, conforme relato de dois casos operados por Ammori et al. ${ }^{19}$ em 2002. A partição laparoscópica também foi posteriormente empregada por Matsumoto et al. em um tumor duodenal obstrutivo em 2005. Por fim, Suzuki et al. ${ }^{20}$ reafirmaram a possibilidade da realização do procedimento por laparoscopia relatando em 2006 uma série de 8 casos operados sem complicações com manutenção da ingestão via oral até o desfecho clínico. Diferentemente de outros autores, Suzuki et al. ${ }^{20}$ propôs a realização da gastro-entero anastomose em "Y de Roux" com o objetivo de evitar sintomas irritativos causados pelo refluxo duodenogastrojejunal de bile para a câmara gástrica proximal.

Ao compararmos a partição gástrica com a gastroentero anastomose verificamos que Kwon et al. ${ }^{14}$ em 2004 encontraram menor perda de peso, menor volume de transfusões sanguíneas e melhor sobrevida em um grupo de 18 pacientes submetidos a partição comparados com um grupo equivalente de pacientes submetidos a gastroentero anastomose. Oida et al. ${ }^{21}$ publicaram em 2009 uma análise retrospectiva comparando 30 pacientes submetidos a gastroenteroanastomose com 30 pacientes submetidos a partição, dentre os quais, cinco casos realizados por laparoscopia. A técnica empregada por Oida et al. ${ }^{21}$ apresentava uma variação pois a partição foi realizada $3 \mathrm{~cm}$ próxima ao tumor seguida de uma nova ressecção triangular da câmara gástrica proximal possibilitando a realização de uma gastro-entero anastomose látero-

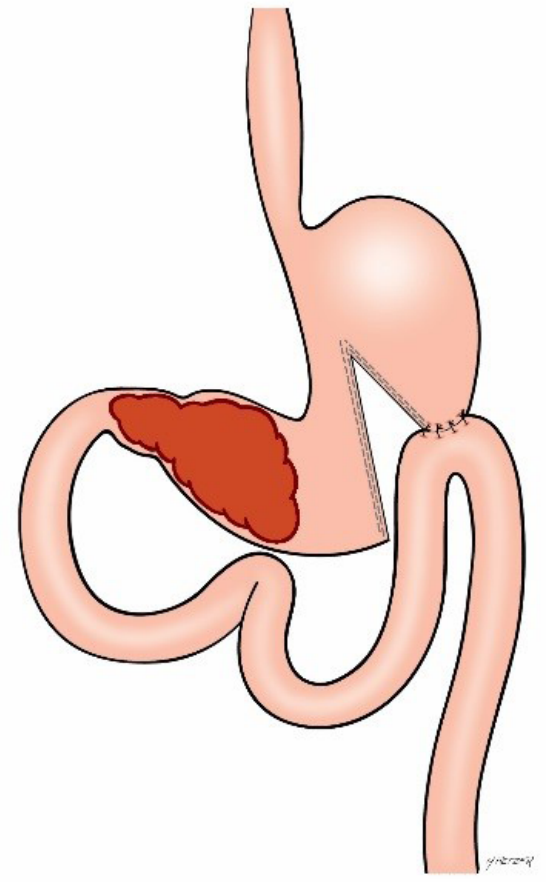

Figura 2. Partição gástrica de Devine modificada

lateral horizontal. Uma anastomose de Braun a $20 \mathrm{~cm}$ da gastro-entero também foi realizada. Os resultados a favor da partição foram expressivos atingindo significância estatística no tempo necessário para a remoção da sonda naso-gástrica ( $\mathrm{SNG}$ ), início da dieta, início da dieta sólida, duração da hospitalização, permanência em casa sem nova hospitalização e tempo de sobrevida. A recidiva da obstrução gástrica não ocorreu em nenhum caso do grupo da partição contra 5 casos $(16,7 \%)$ de nova obstrução que ocorreram no grupo da gastro-entero anastomose.

Frente a estes estudos da literatura, e considerando a elegibilidade dos pacientes e os benefícios que estes teriam, nosso serviço passou a realizar a técnica de partição gástrica em casos pertinentes.

\section{OBJETIVO}

Analisar os resultados operatórios dos pacientes portadores de câncer gástrico avançado distal irressecável e obstrutivo submetidos a esta abordagem cirúrgica paliativa, que é a partição gástrica, em nosso serviço.

\section{PACIENTES E MÉTODOS}

Foram analisados retrospectivamente, em termos de sobrevida e qualidade de vida, os casos de 29 pacientes portadores de adenocarcinoma gástrico distal histologicamente diagnosticado por biópsia com quadro de obstrução gastroduodenal, confirmada com exame de 
imagem ou Endoscopia Digestiva Alta, não candidatos à ressecção cirúrgica e com ausência de outros pontos de obstrução distais à lesão. A análise da qualidade de vida foi baseada no período de hospitalização, tempo necessário para aceitar dieta sólida e manutenção da dieta via oral, já que se trata de uma população de casos graves (sob cuidados paliativos) e para a qual a alimentação por via oral representa uma importante questão.

\section{Intervenção}

A partição gástrica foi realizada de 3 a $5 \mathrm{~cm}$ proximalmente à lesão na grande curvatura utilizando-se grampeador linear cortante (Figuras 3 e 4). O grampeamento foi realizado de maneira horizontal preservando-se um estreito túnel calibrado com Sonda de Faucher $n^{\circ} 32$ na pequena curvatura gástrica (Figuras 5 e 6). Posteriormente, uma gastroentero anastomose, manual ou mecânica, foi realizada de maneira pré-cólica, anisoperistáltica, na parede posterior do estômago com, no mínimo, $5 \mathrm{~cm}$ de extensão utilizando a primeira alça jejunal a cerca de $40 \mathrm{~cm}$ do ângulo de Treitz (Figura 7). Não foi realizada anastomose de Braun.

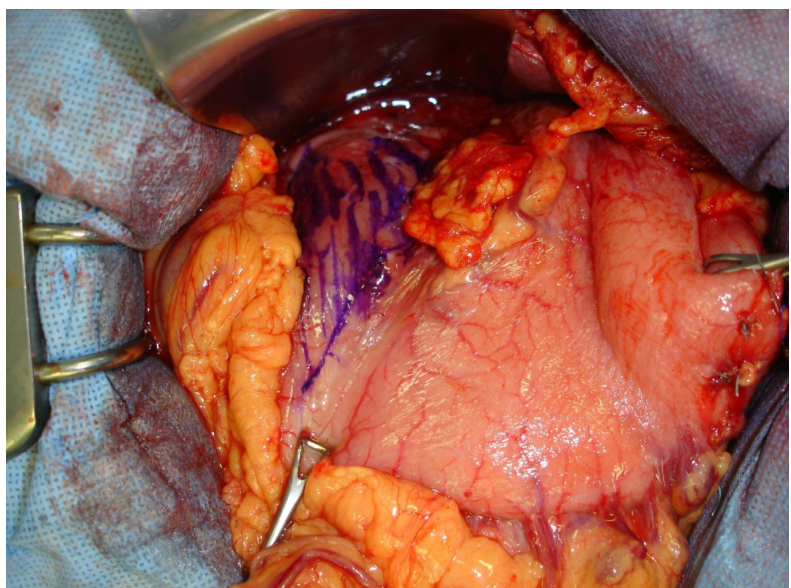

Figura 3. Demarcação do tumor distal

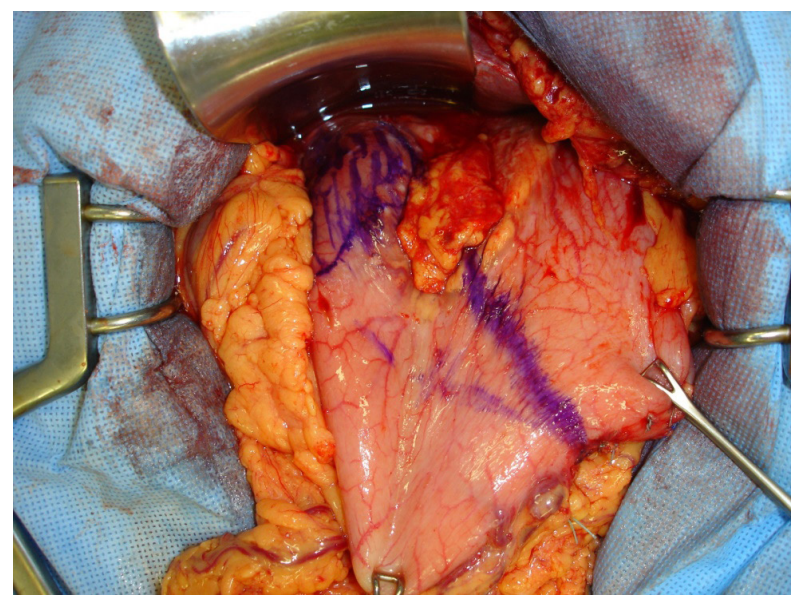

Figura 4. Delimitação do local da partição

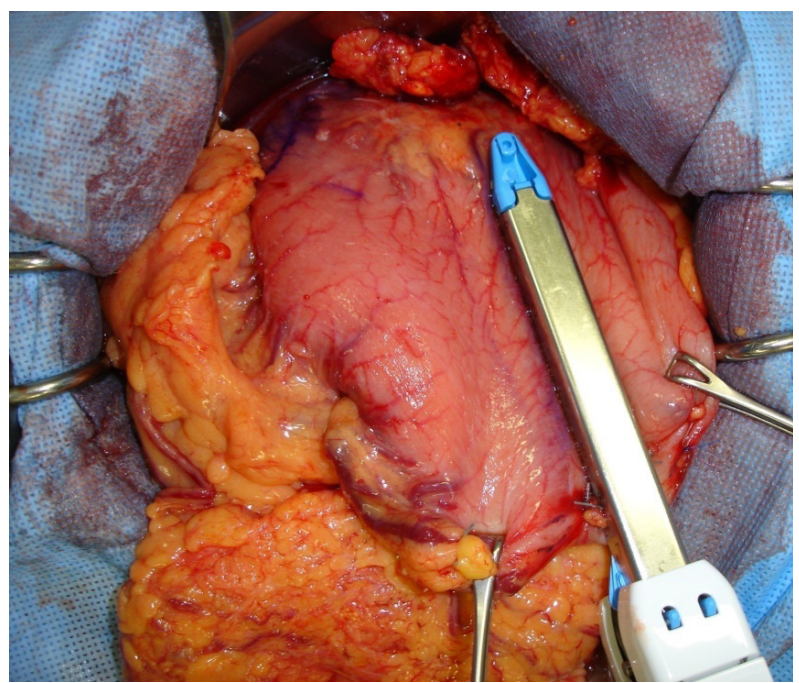

Figura 5. Grampeamento parcial do estômago

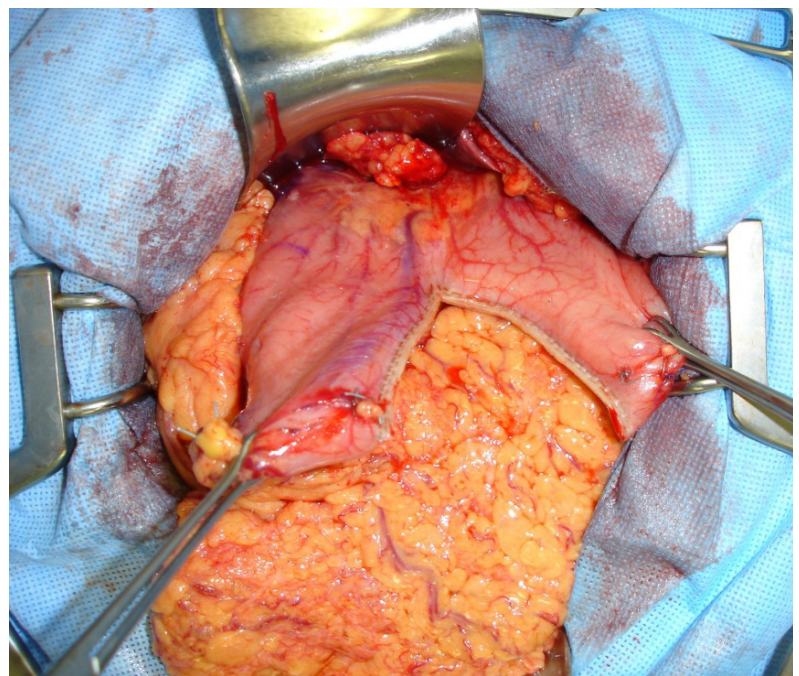

Figura 6. Aspecto pós-partição

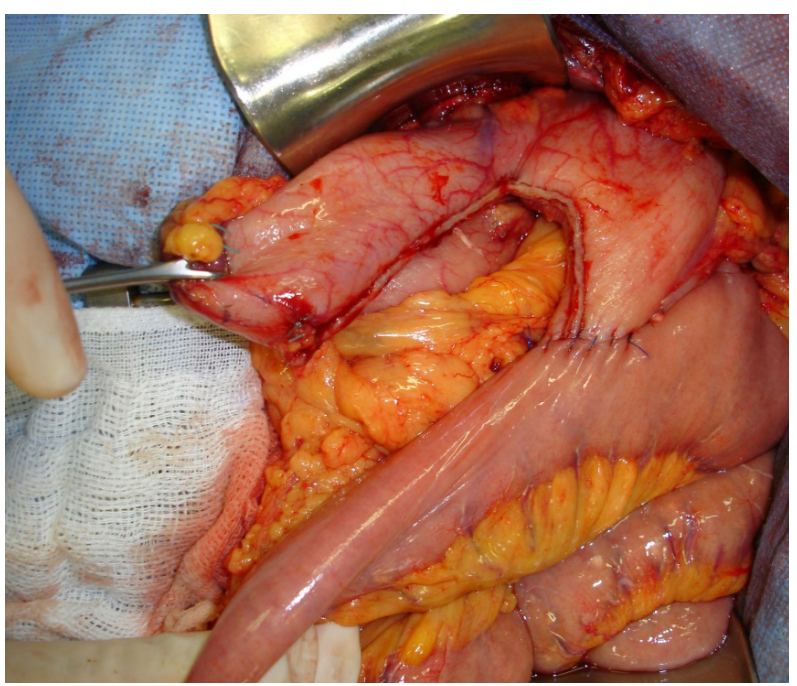

Figura 7. Gastroenteroanastomose na câmara gástrica proximal criada pela partição 


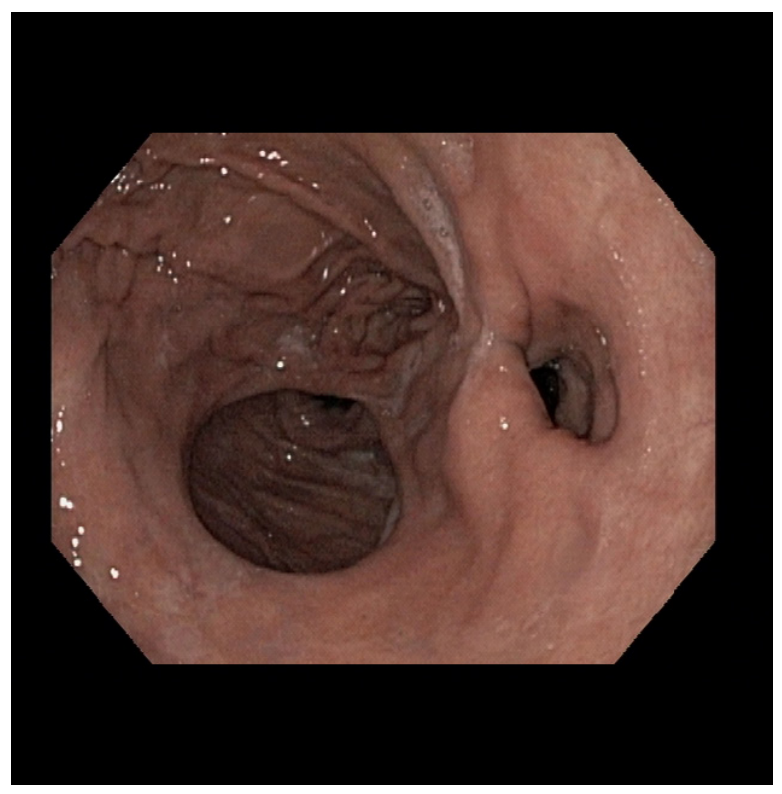

Figura 8. Aspecto endoscópico final, em que se observa a gastroenteroanastomose à esquerda e o conduto pérveo para a câmara gástrica distal (tumoral) à direita

Os pacientes foram mantidos com sonda nasogástrica (SNG), retirada quando o débito diário foi inferior a $300 \mathrm{ml}$ e seguida de introdução de dieta líquida. Os pacientes receberam alta ao aceitar dieta pastosa $(\mathrm{GOSS}=2)$. Para quantificar a intensidade da obstrução gástrica, Adler et al. ${ }^{8}$ propuseram, em 2002, uma classificação denominada GOOSS - Gastric Outlet Obstruction Scoring System - com os seguintes graus:

$$
\begin{aligned}
& 0=\text { ausência de ingestão oral; } \\
& 1=\text { dieta líquida; } \\
& 2=\text { dieta pastosa; } \\
& 3=\text { dieta baixo resíduo ou geral. }
\end{aligned}
$$

Adicionalmente, definimos o retardo do esvaziamento gástrico como impossibilidade de retirada da SNG após três dias, necessidade de reintrodução da SNG ou ausência de deglutição de alimentos pastosos sete dias após o procedimento cirúrgico. Em casos com presença de retardo de esvaziamento gástrico, um exame contrastado do estômago deveria ser realizado.

Os pacientes realizaram seguimento ambulatorial a cada dois meses até o desfecho clínico, e dois pacientes ainda se encontram vivos.

\section{RESULTADOS}

\section{Características da amostra}

Foi estudada uma população de 29 indivíduos, com média de idade de 67,6 anos, sendo 21 pacientes do sexo masculino e 8 do feminino. O IMC (índice de massa corpórea) médio foi de 21,44 e 9 pacientes apresentaram- -se abaixo do peso ou subnutridos (dependendo da idade), com IMC menor que 18,5. O nível médio de hemoglobina foi de $9,53 \mathrm{~g} / \mathrm{dL}$, e $96,3 \%$ dos pacientes se encontravam anêmicos. O nível médio de albumina sérica foi de 3,55 g/ dL e $8 \%$ dos pacientes se encontravam desnutridos (níveis de albumina sérica menores que 3,0 g/dL). Na população de estudo, 53,9\% dos indivíduos apresentavam GOOSS 0 ou 1 (ou seja, com ausência de ingestão alimentar oral ou aceitando apenas dieta líquida) e 100\% apresentavam ECOG performance status ${ }^{22}$ de 0,1 ou 2 (Tabela 2), o que corroborou para a não indicação do tratamento com prótese endoscópica.

Tabela 2. ECOG performance status (Adaptado de: Oken et al. ${ }^{22}$ )

\begin{tabular}{cl}
\hline Classe & ECOG PERFORMANCE STATUS \\
\hline 0 & Totalmente ativo; sem restrições funcionais \\
& $\begin{array}{l}\text { Atividade física estenuante é restrita; deambula sem } \\
\text { qualquer dificuldade e é capaz de realizar trabalho } \\
\text { leve }\end{array}$ \\
& $\begin{array}{l}\text { Capaz de se auto-cuidar, porém incapaz de qualquer } \\
\text { atividade laboral. Capaz de manter-se em pé mais do } \\
\text { que } 50 \% \text { do tempo de vigília }\end{array}$ \\
3 & $\begin{array}{l}\text { Capacidade limitada de auto-cuidados; confinado à } \\
\text { cama ou à cadeira mais de 50\% do tempo de vigília }\end{array}$ \\
4 & $\begin{array}{l}\text { Completamente incapaz, não consegue se auto-cuidar, } \\
\text { totalmente confinado à cama ou à cadeira }\end{array}$ \\
5 & Morto \\
\hline
\end{tabular}

\section{Cursos peri e pós-operatório e sobrevida}

Todos os pacientes da amostra não eram passíveis de terem seus tumores ressecados, por apresentarem carcinomatose peritoneal $(40,7 \%)$, metástase à distância (14,8\%, sendo $100 \%$ destas metástases hepáticas) ou ainda invasão local - estadio T4b - $(70,4 \%)$, em pâncreas (63\%), fígado (14,8\%), mesocólon $(22,2 \%)$, cólon $(3,7 \%)$ e ligamento hepatoduodenal (25,9\%). A duração cirúrgica média foi de 169 minutos e a média de dias de internação foi de 8,6 dias. Em 15 casos, realizou-se o desvio em Y de Roux após a partição, e nos 14 casos restantes, em que não se realizou o desvio, a evolução dos pacientes foi semelhante. Quanto às complicações, utilizamos a classificação de Clavien-Dindo ${ }^{23}$, e houve 4 pacientes em grau II e 2 pacientes em grau V (Tabela 3). Estes dois óbitos representam uma mortalidade operatória de 6,9\%. 
Tabela 3. Classificação de Clavien-Dindo (Adaptado de: Dindo et al. ${ }^{23}$ )

Classificação de Clavien-Dindo (Graus de gravidade das complicações)

Grau I - Pequena alteração no pós-operatório sem necessidade de tratamento farmacológico ou intervenção cirúrgica, endoscópica ou radiológica. Pode incluir antieméticos, antipiréticos, analgésicos, diuréticos, soros e fisioterapia. Inclusão de infecção do local cirúrgico drenado à cabeceira do doente.

Grau II - Necessidade de tratamento farmacológico para além do referido no grau I.

Necessidade de transfusões ou de nutrição parenteral.

Grau III - Necessidade de intervenção cirúrgica, endoscópica ou radiológica.

IIIa - Intervenção sem anestesia geral.

IIIb - Intervenção com anestesia geral.

Grau IV - Complicações graves (incluindo do SNC) a necessitar de UCI ou de Cuidados Intermédios.

IVa - Insuficiência orgânica (incluindo diálise).

IVb - Insuficiência multiorgânica.

Grau V - morte do doente.

Os pacientes demoraram, em média, 3 dias para atingirem GOOSS 1 (aceitação de dieta líquida) e 5 dias para atingirem GOOSS 2 (aceitação de dieta pastosa), no pós-operatório. Ao longo do seguimento, o GOOSS final foi de 2 ou 3 (aceitação de dieta pastosa, de baixo resíduo ou geral) em $96,15 \%$ dos pacientes, e $100 \%$ dos pacientes mantiveram a capacidade de ingestão via oral até o óbito. A sobrevida média da população estudada foi de 213 dias, estando dois pacientes ainda em seguimento na data da submissão deste trabalho.

\section{DISCUSSÃO}

O câncer gastrico avançado irressecável tem se apresentado como um problema cada vez mais frequente em nosso hospital, haja vista que somos um centro de referência para doentes oncológicos. O tratamento paliativo mais tradicionalmente empregado, com a técnica da gastrojejunoanastomose, costuma apresentar resultados insatisfatórios com relação à manutenção prolongada da possibilidade de ingestão via oral do paciente. Nesse contexto, a prótese endoscópica se apresentou como alternativa, porém, em nosso meio, a dificuldade de emprego desta por questões de ordem econômica e a falta de manutençao prolongada da ingesta via oral, acabaram limitando o uso desse método apenas para pacientes com sobrevida de curta duração esperada.
A partição surgiu nesse cenário como uma alternativa. Estudos retrospectivos comparando a gastroenteroanastomose e a partição gástrica ${ }^{14,20,24}$, já demonstraram que a partição apresenta maior sobrevida com uma menor perda sanguínea (por evitar o contato entre alimento e tumor), possibilitando o emprego da terapia quimioterápica paliativa por um periodo mais prolongado de tempo. Em nosso meio, nenhum estudo havia avaliado o emprego da partição. Neste estudo, demonstrou-se que a partição é um procedimento seguro, com baixa taxa de complicações, e com mortalidade baixa, levando-se em consideração a gravidade dos pacientes operados.

Quanto à sobrevida, esta população apresentou uma média de 213 dias, superior à de populações relatadas em estudos como os de Kwon e Lee ${ }^{14}$ (209 dias) e Oida et al. ${ }^{21}$ (192 dias); ainda levando-se em consideração o fato de dois pacientes de nossa população ainda estarem vivos, muito provavelmente esta sobrevida média poderá aumentar.

No que se refere ao tempo de internação, verificouse uma média de 8,6 dias, menor que as apresentadas nesses estudos relatados, que variaram entre 11 e 14 dias. Outro resultado favorável encontrado foi o tempo que os pacientes levaram para aceitar dieta sólida, de 5 dias em nosso estudo, comparado aos 7 dias apresentados pelas populações de Kwon e Lee ${ }^{14}$ e Oida et al. ${ }^{21}$. Além disso, considerando que a maioria dos pacientes $(53,9 \%)$ se apresentavam em GOOSS graus 0 ou 1, que, ao longo do seguimento, $96,15 \%$ atingiram GOOSS final graus 2 ou 3 e que a totalidade da população manteve a capacidade de ingestão via oral até o óbito, a qualidade de vida desses pacientes aumentou.

Entretanto, nosso tempo operatório médio foi de 169 minutos, mais elevado em relação à média obtida no estudo de Oida et al. ${ }^{21}$ (87 minutos). Isso se deveu ao fato de que, muitas vezes, foi tentada a ressecção do tumor no ato operatório até que se estabelessece a irressecabilidade, elevando a duração cirúrgica média. Além disso, infelizmente, quanto à anemia, não dispomos de dados confiáveis para fazer a comparação com os estudos citados e constatar se nossa população precisou de menos transfusões sanguíneas ou não.

Em nossa casuística, em 15 pacientes foi realizado o desvio em Y de Roux após a partição e em 14, apenas a partição com reconstrução do trânsito através da gastroenteroanastomose. Como toda a população apresentou bom resultado com relação à manutenção da dieta via oral, consideramos não ser obrigatório o emprego da técnica em Y de Roux, que, para ser executada, requer a confecção de duas anastomoses (uma a mais do que a partição gástrica apenas), aumentando o tempo operatório. Desta forma, atualmente em nosso serviço optamos por não mais realizar a técnica em Y de Roux quando realizamos a partição.

Frente a estes resultados, no sentido de dar maior credibilidade a este procedimento, nosso serviço está conduzindo um estudo prospectivo randomizado 
comparando as evoluções de pacientes submetidos à gastroenteroanastomose e à partição gástrica.

\section{CONCLUSÃO}

A partição gástrica é uma técnica efetiva e segura

\section{REFERÊNCIAS}

1. Hartgrink HH, Jansen EP, van Grieken NC, van de Velde CJ. Gastric cancer. Lancet. 2009;374(9688):477-90. DOI: http:// dx.doi.org/10.1016/S0140-6736(09)60617-6.

2. Neugut AI, Hayek M, Howe G. Epidemiology of gastric cancer. Semin Oncol. 1996;23(3):281-91.

3. Jeon KS, Chang ES. A clinical analysis of unresectable stomach cancer. J. Korean Surg. Soc. 1991;40:571-80.

4. Lee KK, Kwon SJ. Clinicopathological analysis of unresectable stomach cancer. J Korean Surg Soc. 1999;56:369-77.

5. Ouchi K, Sugawara T, Ono H, et al. Therapeutic significance of palliative operations for gastric cancer for survival and quality of life. J Surg Oncol 1998;69(1):41-4. doi: 10.1002/(SICI)1096-9098(199809)69:1<41::AID$\mathrm{JSO} 8>3.0 . \mathrm{CO} ; 2-\mathrm{K}$.

6. Bozzetti F, Bonfanti G, Audisio RA, et al. Prognosis of patients after palliative surgical procedures for carcinoma of the stomach. Surg Gynecol Obstet. 1987;164:151-4.

7. Stupart DA, Panieri E, Dent DM. Gastrojejunostomy for gastric outlet obstruction in patients with gastric carcinoma. S Afr J Surg. 2006;44(2):52-4.

8. Adler DG, Baron TH. Endoscopic palliation of malignant gastric outlet obstruction using self-expanding metal stents: experience in 36 patients. Am J Gastroenterol. 2002;97:72-8. doi: 10.1111/j.1572-0241.2002.05423.x.

9. Moura EG, Ferreira FC, Cheng S, Moura DT, Sakai P, Zilberstein B. Duodenal stenting for malignant gastric outlet obstruction: prospective study. World J Gastroenterol. 2012;18(9):938-43. doi: 10.3748/wjg.v18.19.938.

10. Jeurnink SM, Steyerberg EW, van Hooft JE, van Eijck CH, Schwartz MP, Vleggaar FP, Kuipers EJ, Siersema PD; Dutch SUSTENT Study Group. Surgical gastrojejunostomy or endoscopic stent placement for the palliation of malignant gastric outlet obstruction (SUSTENT study): a multicenter randomized trial. Gastrointest Endosc. 2010;71(3):490-9. doi: 10.1016/j.gie.2009.09.042.

11. Bresciani CJC, Gama-Rodrigues J, Pinotti HW. Câncer gástrico avançado. Conduta cirúrgica. ABCD Arq Bras Cir Dig. 1990;38:41.

12. Japanese Gastric Cancer Association. Japanese Gastric Cancer Treatment Guidelines 2010 (ver. 3). Gastric Cancer. 2011;14(2):113-23. doi: 10.1007/s10120-011-0042-4.

13. Gastrointestinal Tumor Study Group. The concept of locally advanced gastric cancer. Effect of treatment on outcome. The Gastrointestinal Tumor Study Group. Cancer. 1990;66(11):2324-30. para ser empregada nos casos de pacientes portadores de câncer gástrico avançado distal obstrutivo e irressecável, proporcionando sobrevida, manutenção de dieta via oral e qualidade de vida aos pacientes.

14. Kwon SJ, Lee HG. Gastric partitioning gastrojejunostomy in unresectable distal gastric cancer patients. World J Surg. 2004;28(4):365-8. doi: 10.1007/s00268-003-7212-4.

15. Doberneck RC, Berndt GA. Delayed gastric emptying after palliative gastrojejunostomy for cancer of the pancreas. Arch Surg. 1987;122:827-9. doi: 10.1001/ archsurg.1987.01400190093020.

16. Devine HB. Basic principle and supreme difficulties in gastric surgery. Surg Gynecol Obstet. 1925;40:1-16.

17. Maingot R. The surgical treatment of irremovable cancer of the pyloric segment of the stomach. Ann Surg. 1936;104:161-6. Available from: http://www.ncbi.nlm.nih. gov/pmc/articles/PMC1390229/pdf/annsurg00541-0007. pdf.

18. Kaminishi M, Yamaguchi H, Shimiza N, et al. Stomachpartitioning gastrojejunostomy for unresectable gastric carcinoma. Arch Surg. 1997;132:184-7. doi: 10.1001/ archsurg.1997.01430260082018.

19. Ammori BJ, Boreham B. Laparoscopic devine exclusion gastroenterostomy for the palliation of unresectable and obstructing gastric carcinoma. Surg Laparose Endosc Percutan Tech. 2002;12(5):353-5.

20. Suzuki O, Shichinohe T, Yano T, Okamura K, Hazama K, Hirano S, Kondo S. Laparoscopic modified Devine exclusion gastrojejunostomy as a palliative surgery to relieve malignant pyloroduodenal obstruction by unresectable cancer. Am J Surg. 2007;194(3):416-8. doi: http://dx.doi.org/10.1016/j. amjsurg.2007.02.011.

21. Oida T, Mimatsu K, Kawasaki A, Kano H, Kuboi Y, Amano S. Modified Devine exclusion with vertical stomach reconstruction for gastric outlet obstruction: a novel technique. J Gastrointest Surg. 2009;13(7):1226-32. doi: 10.1007/s11605-009-0874-y.

22. Oken MM, Creech RH, Tormey DC, Horton J, Davis TE, McFadden ET, Carbone PP. Toxicity and response criteria of the Eastern Cooperative Oncology Group. Am J Clin Oncol. 1982;5:649-55.

23. Dindo D, Demartines N, Clavien PA. Classification of surgical complications: a new proposal with evaluation in a cohort of 6336 patients and results of a survey. Ann Surg. 2004;240:205-13. doi: 10.1097/01.sla.0000133083.54934. ae

24. Usuba T, Misawa T, Toyama Y, Ishida Y, Ishii Y, Yanagisawa S, Kobayashi S, Yanaga K. Is modified Devine exclusion necessary for gastrojejunostomy in patients with unresectable pancreatobiliary cancer? Surg Today. 2011;41(1):97-100. doi: 10.1007/s00595-009-4246-8. 